\title{
Hickson 62
}

\section{Kinematics of NGC 4778}

\author{
M. Spavone ${ }^{1}$, E. Iodice ${ }^{2}$, G. Longo ${ }^{1,2,3}$, M. Paolillo ${ }^{1,3}$, and S. Sodani ${ }^{1}$ \\ ${ }^{1}$ Dipartimento di Scienze Fisiche, Universitá Federico II, via Cinthia 6, 80126 Napoli, Italy \\ e-mail: spavone@na.infn.it \\ 2 INAF, Osservatorio Astronomico di Capodimonte, via Moiariello 16, 80131 Napoli, Italy \\ 3 INFN, Sezione di Napoli, via Cinthia 6, Napoli, Italy
}

Received 18 May 2006 / Accepted 30 June 2006

ABSTRACT

\begin{abstract}
Context. Detailed studies of the photometric and kinematical properties of compact groups of galaxies are crucial to understand the physics of galaxy interactions and to shed light on some aspects of galaxy formation and evolution. In this paper we present a kinematical and photometrical study of a member, NGC 4778, of the nearest $(z=0.0137)$ compact group: Hickson 62.

Aims. The aim of this work was to investigate the existence of kinematical anomalies in the brightest group member, NGC 4778 in order to constrain the dynamical status and the formation history of the group.

Methods. We used long-slit spectra obtained with FORS1 at VLT, to measure line-of-sight velocity distributions by means of the Fourier Correlation Quotient method, and to derive the galaxy rotation curve and velocity dispersion profile.

Results. Our analysis reveals that Hickson 62a, also known as NGC 4778, is an S0 galaxy with kinematical and morphological peculiarities, both in its central regions $\left(r<5^{\prime \prime}\right)$ and in the outer halo. In the central regions, the rotation curve shows the existence of a kinematically decoupled stellar component, offset with respect to the photometric center. In the outer halo we find an asymmetric rotation curve and a velocity dispersion profile showing a rise on the SW side, in direction of the galaxy NGC 4776.

Conclusions. The nuclear counterrotation, the distorted kinematics in the outer halo and the X-ray properties of the group suggest that NGC 4778 may be the product of a recent minor merger, more reliable with a small late-type galaxy.
\end{abstract}

Key words. galaxies: kinematics and dynamics - galaxies: evolution - galaxies: interactions - galaxies: clusters: general

\section{Introduction}

Poor groups of galaxies are the most common cosmic structures and contain a large fraction of the galaxies present in the universe (Tully \& Fisher 1987; Eke 2004). At a difference with rich clusters, they span a wide range of densities, from loose groups, having spatial density of baryonic matter slightly above that of the field, to compact ones having densities comparable or higher than those encountered in the cores of the richest clusters. For this reason, they are the ideal ground where to test all scenarios for galaxy formation and evolution and where to pinpoint the details of the physics controlling galaxy interactions.

Loose groups having masses in the range $10^{13}-10^{14} M_{\odot}$, almost certainly are still collapsing and are therefore crucial to uncover the formation processes shaping cosmic structures (Zabludoff \& Mulchaey 1998). Many factors converge in identifying compact groups as good candidates to be one of the regions where some of this processes occur. In first place, their high spatial density of luminous matter and small velocity dispersions imply dynamical lifetimes of the order of a fraction of the Hubble time. This leading to the possibility that the groups observed in the present time and in the local universe are second generation objects, just accreting new members from the loose groups of galaxies in which almost always they are embedded (Vennik et al. 1993). Second, compact groups are numerous and contain a non negligible fraction of the baryonic matter in the nearby universe (Pildis et al. 1996). Therefore, whatever is their ultimate fate, they are bound to have an impact on the observable properties of galaxies and cosmic structures.

As it was stressed in Mendes de Oliveira et al. (2003, hereafter M03), the influence of environmental effects on the internal dynamics and matter distribution of compact group galaxies has not yet been clearly established, mostly due to lack of reliable kinematic data. Extensive kinematical studies of both the stars and gas in galaxies belonging to compact groups (Rubin et al. 1991; Nishiura et al. 2000; M03; Rampazzo et al. 1998; Bonfanti et al. 1999) all suggest that peculiar kinematical behaviors are much more common $(\sim 75 \%)$ than in the field.

Moreover, M03 showed that velocity fields of the ionized gas component in galaxies belonging to compact groups are often significantly affected by non-circular motions, local asymmetries and misalignments between the kinematic and stellar axes. These peculiarities, however, tend to smooth out if the rotation curve is derived by averaging the velocity fields of the galaxies over large regions. If these averaged values are used, a large fraction $(\sim 80 \%)$ of the HCG members follow the same TullyFisher (TF) relationship of field galaxies (M03). This may indicate that the haloes of compact group galaxies have not been significantly stripped inside their optical size. However, according to M03, the remaining $20 \%$ of the galaxies, including the lowest-mass systems, present significant anomalies which could be explained by assuming that compact group galaxies have smaller dark halos than their field counterparts, due to tidal truncation. A result which finds support in numerical simulations 
Table 1. Summary of the properties of the Hickson 62 members. Data are taken from Nasa/IPAC Extragalactic Database.

\begin{tabular}{lcccc}
\hline \hline & NGC 4778 (HCG62a) & NGC 4776 (HCG62b) & NGC 4761 (HCG62c) & NGC 4764 (HCG62d) \\
RA $_{\mathrm{J} 2000}$ & 125306 & 125305 & 125310 & 125306.6 \\
$\delta_{J 2000}$ & -091214 & -091200 & -091155 & -091528 \\
Type & $\mathrm{S} 0$ & $\mathrm{~S} 0$ & $\mathrm{~S} 0$ & $\mathrm{E} 2$ \\
Size $(\operatorname{arcsec})$ & $52.3 \times 34.3$ & $44.2 \times 33.3$ & $24.6 \times 12.3$ & $19.9 \times 16.8$ \\
Redshift & 0.0142 & 0.01188 & 0.01478 & 0.01392 \\
$m_{v}$ & 13.47 & 14.04 & $14 . .86$ & 15.98 \\
$m_{\mathrm{b}}$ & 13.79 & 14.21 & 15.00 & 16.30 \\
$m_{r}$ & 11.25 & 12.04 & 13.59 & 14.11 \\
Ext. & 0.227 & 0.227 & 0.224 & 0.217 \\
\hline
\end{tabular}

(cf. Governato et al. 1991) and has important consequences on the groups dynamical lifetimes.

In spite of the vast literature existing, due both to the limited statistics and to the problems encountered in disentangling true groups from optical ones as well as in deprojecting the measured kinematic and photometric quantities, our understanding of the dynamical and evolutionary status of compact groups still presents quite a few gaps. Gaps which can be filled only through detailed multitechnique and multiwavelength analysis of individual cases. In this respect, the dynamical and evolutive status of a group two observables are crucial: the detailed kinematics of the individual galaxies and the structure of the diffuse hot gas halo. In this paper and in Paper II (Sodani et al. 2006) we present a study of the compact group of galaxies Hickson 62 based on archival optical, spectroscopic and X-ray data extracted from the ESO and the Chandra archives.

In this first paper we focus mainly on the peculiar kinematics and on the photometry of the dominant galaxy NGC 4778 (Hickson 62a), while in Paper II we shall discuss the diffuse $\mathrm{X}$-ray halo embedding at least two of the group components. This paper is structured as follows: in Sect. 2 we discuss the main characteristics of Hickson 62, in Sect. 3 we describe the observations and the data reduction procedure. The photometric properties of NGC 4778 are presented in Sect. 4 and the kinematics in Sect. 5. Finally, we draw our conclusions in Sect. 6. Throughout this paper we shall adopt a distance of $60.9 \mathrm{Mpc}$ based on $H_{0}=70 \mathrm{~km} \mathrm{~s}^{-1} \mathrm{Mpc}^{-1}$ and an heliocentric radial velocity $V=4260 \mathrm{~km} \mathrm{~s}^{-1}$, this implies $1 \operatorname{arcsec}=0.29 \mathrm{kpc}$.

\section{HCG62}

Hickson 62 is a quartet of accordant early type galaxies at a redshift of 0.0137 (Hickson et al. 1988). It also belongs to the loose group LGG 313 (Rood \& Struble 1994; Tovmassian 2001) containing at least 13 galaxies within a distance of $200 \mathrm{kpc}$. The group is dominated by the pair NGC 4778 (also known as HCG 62a) and NGC 4776 (HCG 62b), having a projected separation of only $8 \mathrm{kpc}$. NGC 4778 formerly classified as an E3 galaxy, is now classified as an S0 with a bright compact nucleus and subsequently discovered to be a low luminosity AGN (Fukazawa et al. 2001; and Coziol et al. 1998). Both NGC 4776 and NGC 4761 (HCG62c) are classified as peculiar S0's. Finally, NGC 4764 (HCG62d) is a faint E2 galaxy slightly more distant from the center of the action. The main photometric and morphological characteristics of the group galaxies are summarized in Table 1.

The compact group is also embedded in a bright X-ray halo which extends out to $200 \mathrm{kpc}$, revealing the presence of a deep common gravitational well centered on NGC 4778. The high resolution Chandra images also showed the presence of large cavities in the gaseous halo due to the interaction of relativistic plasma with the hot IGM, a sign of recent activity due to the NGC 4778 central AGN (Vrtilek et al. 2002).

NGC 4778 has been the target of several studies. Long slit spectroscopy has been obtained at different position angles. Bettoni et al. (1995) and Rampazzo et al. (1998), positioned the slit along the line joining the nuclei of NGC 4778 and NGC 4776 $\left(\mathrm{PA}=127^{\circ}\right)$ and their results lead to the conclusion that the pair NGC 4778/4776 is not interacting and the kinematical peculiarities observed in NGC 4778 are likely due to an interaction with NGC 4761. In fact, the velocity dispersion and rotation curves of NGC 4776 are well behaved and appear unperturbed, while the velocity dispersion profile of NGC 4778 shows a relatively sharp increase to the SE, suggestive of the presence of a perturber.

\section{Observations and data reduction}

\subsection{Spectroscopic data}

The spectroscopic data were extracted from the European Southern Observatory (ESO) public archive ${ }^{1}$. They have been obtained with the FORS1 spectrograph at VLT-UT1. The detector is a $2048 \times 2048$ pxl Tektronix CCD, with a scale of $0.2^{\prime \prime}$ pixel $^{-1}$ (with the standard resolution collimator). The data, consisting in four set of spectra, were acquired with a slit $1.6^{\prime \prime}$ wide and 6.8 long, using the GRIS-600V grism with a dispersion of $49 \AA \mathrm{mm}^{-1}$, corresponding to $1.18 \AA \mathrm{pxl}^{-1}$, in the 4650-7100 $\AA$ wavelength range. The spectra were acquired along the photometric major axis $\left(\mathrm{PA}=80^{\circ}\right)$ of NGC 4778 and, by chance, they also intercepted NGC 4761 in a direction parallel to its minor axis, slightly offcentered to SE side with respect to the nucleus (see Fig. 1). The total integration time of the spectra is $2700 \mathrm{~s}$ and the average seeing turned out to be $1^{\prime \prime}$. A set of spectra of standard template $F$ stars, were also acquired with the same configuration.

Individual frames were pre-reduced using the standard MIDAS image processing package; the wavelength calibration was made using the IRAF TWODSPEC.LONGSLIT package and a set of He-Ar-Ne lamp spectra, taken for each observing night. The spectral resolution turned out to be $2.8 \AA$ (FWHM), equivalent to a velocity resolution of $\sigma \simeq 60 \mathrm{~km} \mathrm{~s}^{-1}$. Sky subtraction was performed using a narrow region at both edges of the slit where there was minimum galaxy contamination. Finally, all exposures were co-added in a final median averaged 2D spectrum (cf. Fig. 2). The final steps of spectral processing consisted of $i$ ) binning the spectra along the spatial direction in order to achieve a signal-to-noise $(S / N) \geq 50$ at all radii (which is the $S / N$ measured at the last data points, while the central pixels have $S / N$ about 3 times larger), leaving no more than 2 data points within the seeing disk; ii) removing the galaxy continuum

\footnotetext{
${ }^{1}$ http://archive.eso.org/
} 


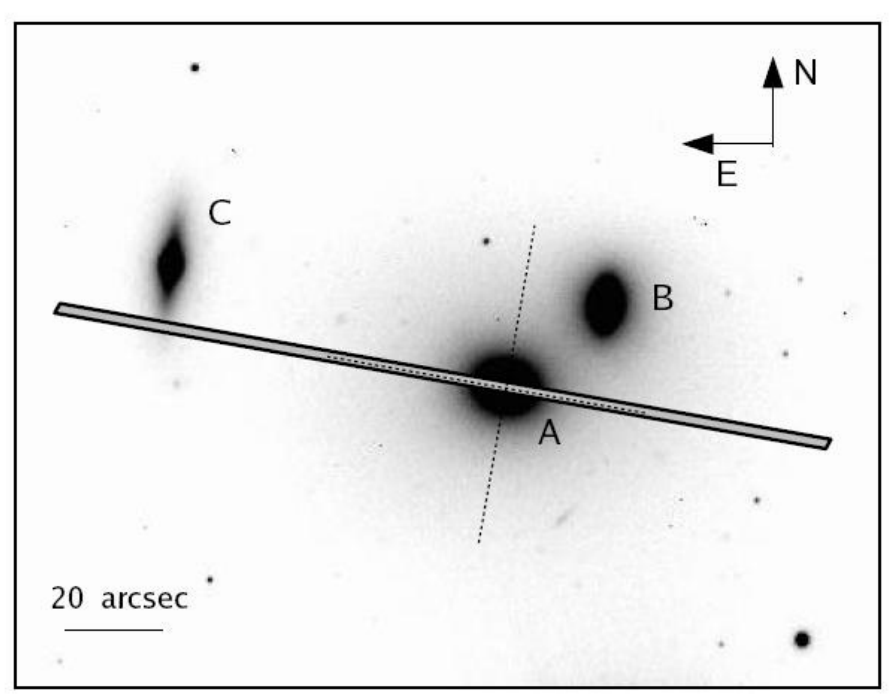

Fig. 1. $B$ band image of HCG62 with the slit overlayed. The dashed lines overlayed on the slit represent the directions along which we have extracted the light profiles presented in Fig. 5.

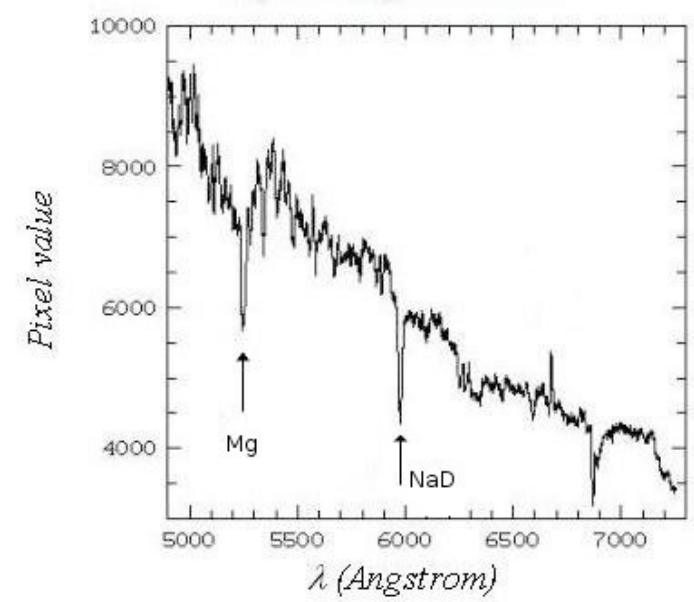

Fig. 2. Observed spectrum of NGC 4778. The main absorption features used for the kinematical analysis are marked.

by fitting a fourth order polynomial (for a detailed description of the procedures see Bender et al. 1994).

\subsection{Imaging data for NGC 4778}

We also extracted from the ESO archive CCD images of Hickson 62 obtained with FORS1 at ESO VLT-UT1 in the Jonson $B$ and $R$ bands. The CCD was the same used for the spectra, and the exposure times were $240 \mathrm{~s}$ and $180 \mathrm{~s}$ in the $B$ and $R$ band, respectively. All images were taken in fairly good (i.e. $F W H M \simeq 1^{\prime \prime}$ ) seeing conditions. The raw CCD frames were pre-processed using the MIDAS image processing package and standard techniques for de-biasing and flat-fielding. Unfortunately, standard stars were not available to perform absolute photometric calibration and we were forced to adopt the tabulated zero point, namely: $27.594 \pm 0.045$ and $27.939 \pm 0.025$ for the $B$ and $R$ band, respectively. We used the IRAF-ELLIPSE task on the $B$ band image to perform the isophotal analysis, to derive the effective parameters (the effective radius turns out to be $R_{\mathrm{e}}=13 \pm 1$ arcsec), and to derive the diskyness parameter, ellipticity and position angle for the NGC 4778 isophotes.
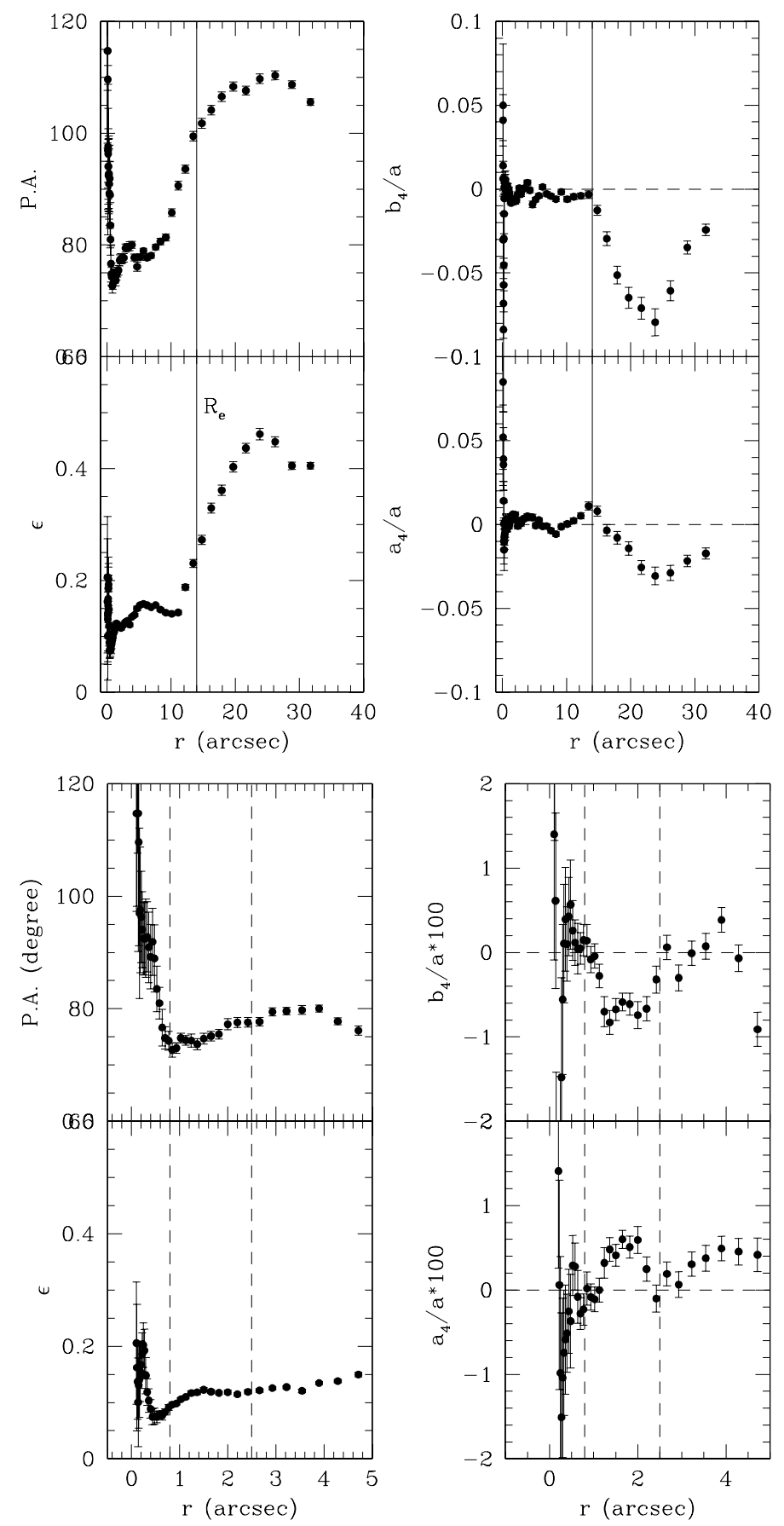

Fig. 3. Top panel - Ellipticity, position angle (PA) and diskiness for NGC 4778. The solid line indicates $R_{\mathrm{e}}$. The trends of all parameters are different from those of $r<R_{\mathrm{e}}$. Bottom panel - The same as above for the nuclear region of NGC 4778. The first dashed line indicates the limit over which the data points are not affected by the seeing. The second dashed line marks the region discussed in the text.

\section{Photometry of NGC 4778}

Here we describe and discuss the main features in the light distribution of NGC 4778, in order to better understand the kinematical properties described in Sect. 5 and to examine some possible connection between photometry and kinematics.

In Fig. 3 (top panel) we show the results of the isophotal analysis of NGC 4778. For $2^{\prime \prime} \leq r \leq 12^{\prime \prime}$, the ellipticity $(\epsilon)$, Position Angle (PA) and diskyness $\left(a_{4} / a\right)$ are approximately constant, thus indicating that in the intermediate regions 


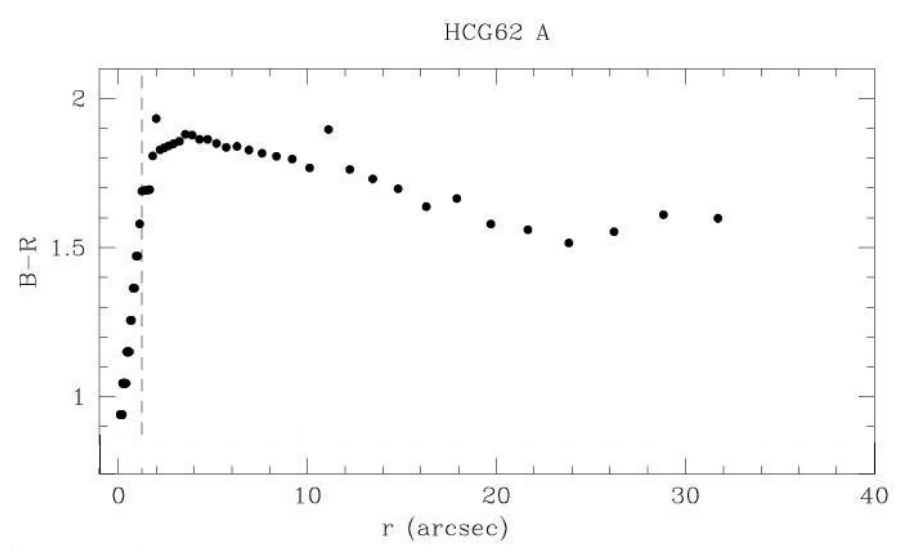

Fig. 4. Mean $B-R$ color profile of NGC 4778. The dashed line indicates the limit of reliability of the photometry.

of the galaxy the isophotes are almost round, co-axial and do not significantly deviate from purely elliptical shape (Bender et al. 1994) At larger radii (for $r \geq 12^{\prime \prime} \sim R_{\mathrm{e}}$ ), isophotes present increasing flattening and become more boxy (i.e. $a_{4} / a<0$ ). Also, the PA changes of about $\sim 30^{\circ}$. These results are consistent with those of Mendes de Oliveira (1992). Looking in more detail the nuclear regions (Fig. 3 bottom panel), between $1^{\prime \prime} \leq r<2.5^{\prime \prime}$ (outside the seeing disk), a small twisting $\left(\sim 10^{\circ}\right)$ and an increasing flattening is observed. In the same regions $a_{4} / a$ is significantly larger than zero.

In Fig. 4 we show the average $B-R$ color profile of NGC 4778. The galaxy has bluer colors in the central regions and, more precisely, we note an inversion of the trend of the color profile beyond $5^{\prime \prime}$. Even though colors may be affected by systematic errors due to the adopted calibration zero point, the mean color profile of NGC 4778 is consistent with the range of values typical for early-type galaxies $(1.65 \leq B-R \leq 1.8$, Fukugita et al. 1995) and for spheroidal galaxies in compact groups $(0.9 \leq B-R \leq 1.7$ for the Es and $1.5 \leq B-R \leq 1.8$ for the S0; see Zepf et al. 1991).

\subsection{The model of the luminosity distribution of NGC 4778}

We performed the 2-dimensional model of the NGC 4778 light distribution through the super-position of a spheroidal central component and an exponential disk (Iodice et al. 2001; Byun \& Freeman 1995). The projected light of the spheroidal component follows the generalized de Vaucouleurs law (Caon et al. 1993):

$\mu_{\mathrm{b}}(x, y)=\mu_{\mathrm{e}}+k\left[\left(\frac{r_{\mathrm{b}}}{R_{\mathrm{e}}}\right)^{1 / n}-1\right]$

with $k=2.17 n-0.355, r_{\mathrm{b}}=\left[x^{2}+y^{2} / q_{\mathrm{b}}^{2}\right]^{1 / 2}$, while $q_{\mathrm{b}}, \mu_{\mathrm{e}}$ and $R_{\mathrm{e}}$ are the apparent axial ratio, the effective surface brightness and the effective radius respectively. The projected light distribution of the exponential disk (Freeman 1970) is given by

$\mu_{\mathrm{d}}(x, y)=\mu_{0}+1.086\left(\frac{r_{\mathrm{d}}}{r_{\mathrm{h}}}\right)$

with $r_{\mathrm{d}}=\left[x^{2}+y^{2} / q_{\mathrm{d}}^{2}\right]^{1 / 2}$, and where $q_{\mathrm{d}} \mu_{0}$ and $r_{\mathrm{h}}$ are the apparent axial ratio, the central surface brightness and the scalelength of the disk respectively.

We used: i) a model made by a bulge-like component with a Sersic law $r^{1 / n}$; ii) a model made by the superposition of a bulgelike component with an $r^{1 / n}$ light distribution, and an exponential

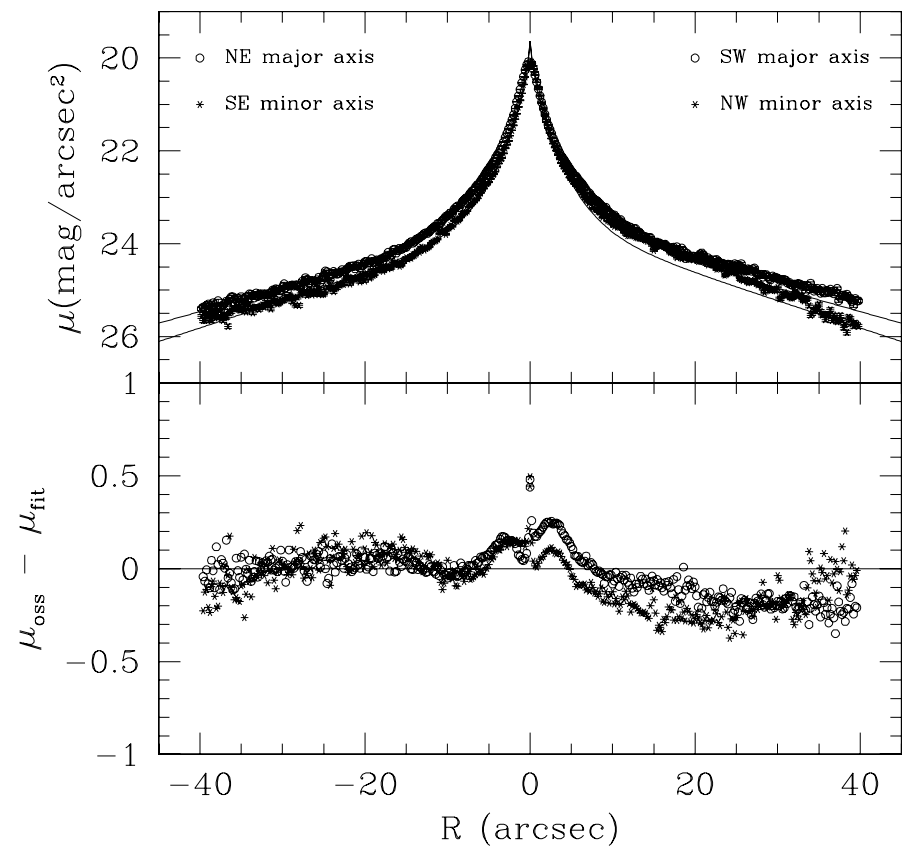

Fig. 5. Top panel - 2D fit of NGC 4778 light distribution. The observed light profiles along the major (open circles), and minor axis (asterisk), are compared with those derived by the fit (continuous line). Bottom panel - Residuals between the observed and the fitted light profiles.

disk. To take into account the systematic effect of the seeing that influence the model parameters, we masked the central area of the galaxy (within the seeing).

The better agreement of the second model with the data, strongly supports the conclusion that NGC 4778 is a misclassified S0 galaxy. The structural parametres derived from the fit are: $\mu_{\mathrm{b}}=23.48 \pm 0.01, r_{\mathrm{h}}=21.9 \pm 0.2 \operatorname{arcsec}, \mu_{\mathrm{e}}=22.55 \pm 0.01$, $r_{\mathrm{e}}=4.60 \pm 0.04$ and $n=1.49 \pm 0.01$, leading to a bulge-to-disk ratio, $L_{\mathrm{B}} / L_{\mathrm{D}} \simeq 0.24$.

Figure 5 shows the comparison between the observed and calculated light profiles in the $B$ band. Residuals show that, in the $\mathrm{SE}$ and NE directions the fitted profiles are in agreement with the observed one. In the NW and SW directions instead, the residuals show that the observed light profile is brighter than the fitted one, due to light contamination from NGC 4776. Notice that the region between $1^{\prime \prime}$ and $5^{\prime \prime}$ from the center, the fit does not reproduce the observed light profile thus suggesting the presence of an additional component for which the light distribution does not follow an $r^{1 / n}$ law (Jog et al. 2006).

\section{Line-Of-Sight Velocity Distribution of HCG62}

The Line-Of-Sight Velocity Distribution (LOSVD) was then derived from the continuum-removed spectra using the Fourier Correlation Quotient (FCQ) method (Bender 1990; Bender et al. 1994). We assumed the LOSVD to be described by a Gaussian function and derived the line-of-sight rotational velocity $v$ and the velocity dispersion $\sigma$. Main sources of statistical and systematics errors are the template mismatching and inaccurate continuum removal (the problem of error estimate was analyzed in detail by Bender et al. 1994; Mehlert et al. 2000; and Gerhard 1993). The errors on each kinematic data-point were derived from photon statistics and CCD read-out noise and were calibrated via Monte Carlo simulations: noise is added to the template star, it is broadened according to the observed values of $\sigma$, 

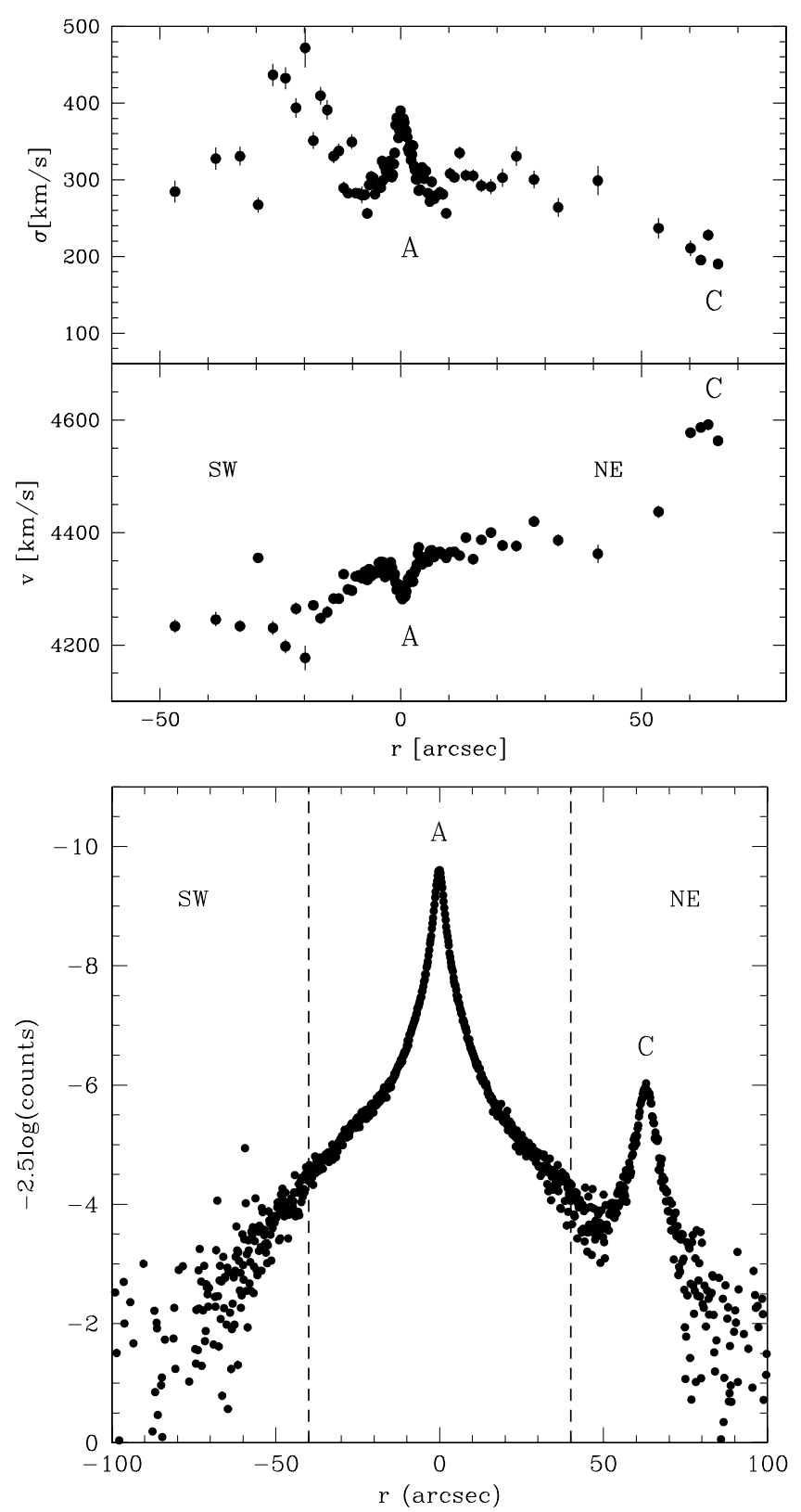

Fig. 6. Top panel - Rotation curve and velocity dispersion profile derived for the whole slit length, which include both galaxies NGC 4778 and NGC 4761 (labelled on the plot as A and C respectively). Bottom panel - Uncalibrated light profile through the slit, which includes both galaxies NGC 4778 and NGC 4761; the dashed lines indicates the region of the spectrum used to derive the kinematics.

the output kinematical values were compared with the input ones.

In Fig. 6 (top panel) we show the line-of-sight radial velocity curve and the velocity dispersions profile for both NGC 4778 and NGC 4761. As the uncalibrated light profile through the slit (see Fig. 6 bottom panel) also shows, the whole kinematic profile extends up to about $60 \operatorname{arcsec}(\sim 17 \mathrm{kpc})$ from the galaxy center, which is about $4 R_{\mathrm{e}}$ and the contribution of NGC 4761 to the whole spectrum is always about $20 \%$. For the study of NGC 4778 we therefore extracted a region in the spectra of 50 arcsec from the galaxy center.

HCG62 A

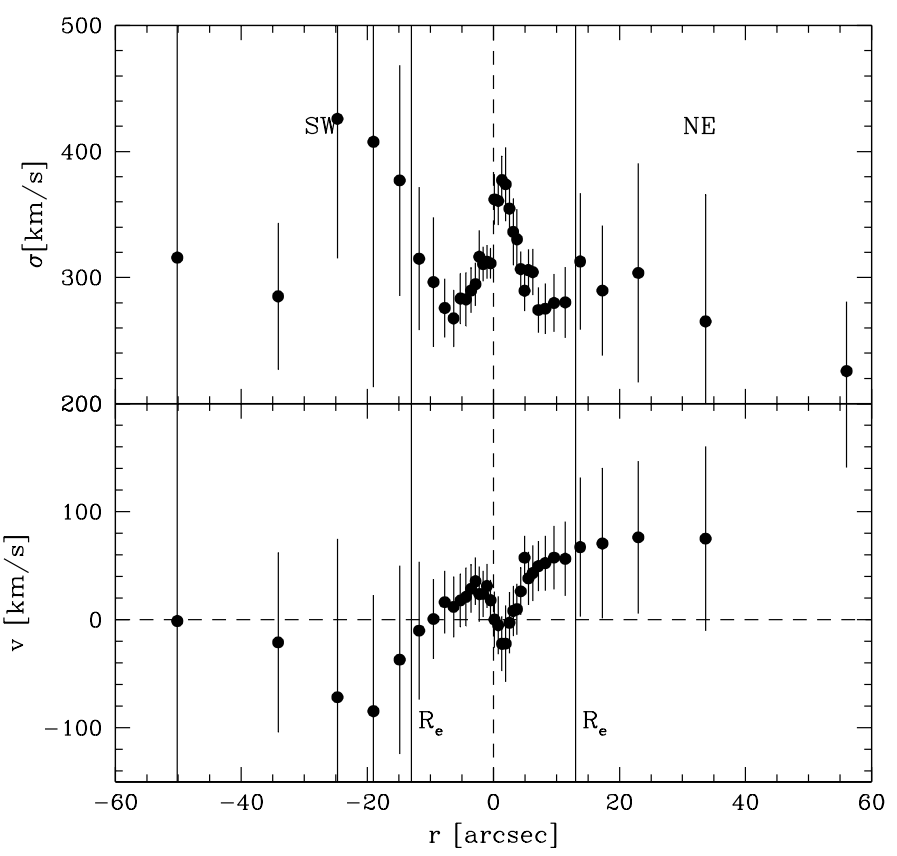

HCG62 A - nuclear region

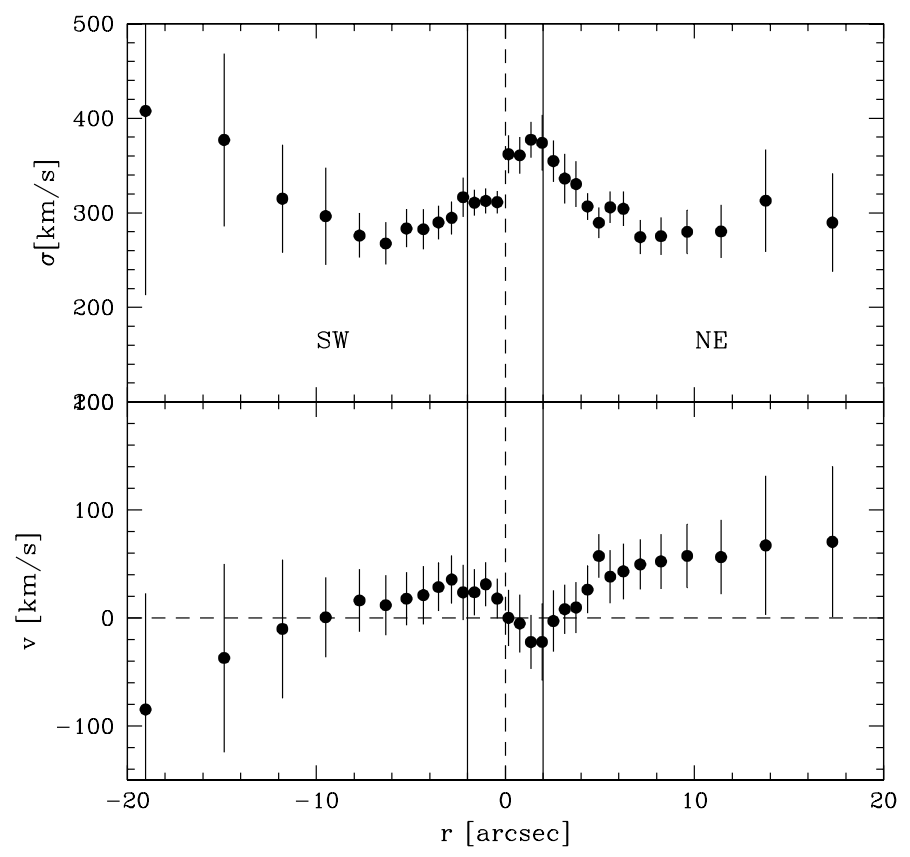

Fig. 7. Rotation curve and velocity dispersion profile derived for NGC 4778 for the whole galaxy extension (top panel) and for the nuclear regions (bottom panel) The vertical solid lines marks the regions discussed in the text.

\subsection{Kinematics of NGC 4778}

The rotation curve and the radial velocity dispersion profile along the major axis of NGC 4778 are shown in Fig. 7. The kinematic profiles reveal two important features: $i$ ) a counter-rotation in the nuclear region, for $r \leq 2.5^{\prime \prime}$; ii) at large galactocentric distances (for $r>2.5^{\prime \prime}$ ) the rotation curve and velocity dispersion are asymmetric with respect to the galaxy center. Note that the central value is chosen in order to obtain the rotation curve symmetric within $3^{\prime \prime}$. Given that, the dynamical center of the 


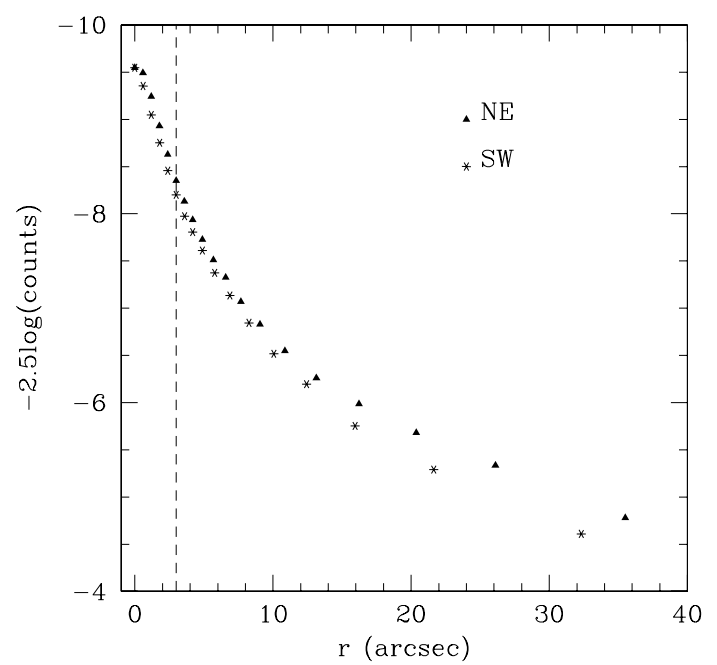

Fig. 8. Comparison of the uncalibrated light profile along the slit in the $\mathrm{NE}$ and SW directions. The data-points were binned to reach a minimum signal-to-noise of 50 .

curves does not correspond to the photometric one, the point of maximum signal to noise ratio, which is offset by $2^{\prime \prime}$ in the NE direction.

On the whole, the galaxy presents significant rotation. An increasing rotation is measured starting from $r \sim 3^{\prime \prime}$ : on the NE side, the rotation reaches a value of about $70 \mathrm{~km} \mathrm{~s}^{-1}$ at $r \sim 13^{\prime \prime}\left(\sim R_{\mathrm{e}}\right)$ and then it remain nearly constant out to about $35^{\prime \prime}\left(\sim 2 R_{\mathrm{e}}\right)$. At larger radii, as we have already seen in the last section, the spectrum is contaminated by the light coming from the galaxy NGC 4761, therefore the value of $200 \mathrm{~km} \mathrm{~s}^{-1}$ detected for $r=55^{\prime \prime}$ cannot be due to the typical motion of the stars in NGC 4778. On the SW side, the velocity increases reaching its maximum value of $80 \mathrm{~km} \mathrm{~s}^{-1}$ at $r \sim 20^{\prime \prime}\left(\sim 1.4 R_{\mathrm{e}}\right)$; at larger radii we observe a drop in the velocity which becomes consistent with zero at $r>35^{\prime \prime}\left(\sim 2.5 R_{\mathrm{e}}\right)$.

The velocity dispersion decreases from the maximum value of about $380 \mathrm{~km} \mathrm{~s}^{-1}$ in the center of the galaxy, to a value of $280-300 \mathrm{~km} \mathrm{~s}^{-1}$ for $r>5^{\prime \prime}$, remaining nearly constant, within the errors, up to $2 R_{\mathrm{e}}$. Both on the $\mathrm{SW}$ and on the NE side, for $3^{\prime \prime} \leq r \leq 5^{\prime \prime}$, the velocity dispersion decreases from $380 \mathrm{~km} \mathrm{~s}^{-1}$ to $280-300 \mathrm{~km} \mathrm{~s}^{-1}$ at $r \sim 5^{\prime \prime}$. At larger radii, the velocity dispersion increases on the SW side, and reaches the maximum value of about $420 \mathrm{~km} \mathrm{~s}^{-1}$ (at $r \sim 20^{\prime \prime}-25^{\prime \prime}$ ), and then decreases reaching values consistent with those observed on the NE side.

Looking in more detail the nuclear region, for $r \leq 2.5^{\prime \prime}$ we detect an inversion of the velocity gradient towards the center, with a maximum value of the velocity of $20-30 \mathrm{~km} \mathrm{~s}^{-1}$, with respect to the outer radii. This inversion within the central regions with respect to the overall trend, reveals the presence of a counter-rotating decoupled core. In correspondence with the inversion of the velocity gradient, the velocity dispersion profile shows an hint for a local minimum (see Fig. 7 bottom panel).

\subsection{Photometry versus kinematics}

In Fig. 8 we plot the comparison of the light profile along the slit in the NE and SW directions, where data-points were binned to reach a minimum signal-to-noise of 50. For $r>3^{\prime \prime}$, where the kinematic shows a clear asymmetry, the light profile along the slit results unperturbed. This suggests that the peculiarities in the outer kinematical properties may be attributed to perturbed motion of the stars in NGC 4778, rather than to a contamination by the light from surrounding galaxies. In particular, on the SW side, the increase detected at $r \sim 20^{\prime \prime}$ in both the rotation curve and the velocity dispersion profile, is likely due to dynamical effects rather than to a projection effect. Gravitational interactions, in fact, lead to the distorsion of the orbits and a tidal heating of the stars surrounding the interacting objects (cf. Combes et al. 1995). For $r \geq 12^{\prime \prime} \sim R_{\mathrm{e}}$, where the kinematic profiles on the SW side also shows abrupt variations, we detect an increasing flattening and twisting of the isophotes.

In order to check whether the observed flattening could be attributed to the rotation or not, we evaluated the anisotropy parameter $(v / \sigma)^{*}$. The anisotropy parameter $(v / \sigma)^{*}$ is defined as the ratio between the observed value of $(v / \sigma)$ and the theoretical value for an isotropic oblate rotator $(v / \sigma)_{t}=[\epsilon /(1-$ $\left.\epsilon)^{1 / 2}\right]$ (Binney 1978), where $\epsilon$ is the observed ellipticity. For NGC 4778, at $r \sim 2 R_{\mathrm{e}}\left(\sim 30^{\prime \prime}\right)$, where $\epsilon \sim 0.4$, we estimate $(v / \sigma)^{*} \sim 0.66$. Comparing this result with those obtained by Bender et al. (1994) for a sample of 44 elliptical galaxies, we find that in the $M_{B}-\log (v / \sigma)^{*}$ plane ${ }^{2}$, NGC 4778 is located among the rotationally supported galaxies (see Fig. 18 of Bender et al. 1994).

In the nuclear regions, where kinematic shows a decoupled component with an inversion of the velocity gradient, we have $a_{4} / a>0$, which indicates the presence of disky isophotes (see Fig. 3 bottom panel).

\section{Discussion and conclusion}

We have analyzed high signal-to-noise spectra along the major axis of the dominant galaxy of the Hickson Compact Group HCG62. On the whole, the observed kinematics and photometry of NGC 4778 is consistent with that of an S0 galaxy.

- Nuclear regions. The higher resolution data enabled us to detect the signature of the nucleus of NGC 4778: inside $3^{\prime \prime}$ we observe an inversion in the velocity profile gradient, which also correspond to some anomalous photometric features, such as bluer colors, and twisting in the position angle of the isophotes. These features strongly suggest the existence of a small core ( $\sim 600$ pc) kinematically decoupled from the whole galaxy. Such anomalous Kinematically Decoupled Cores (KDC) are common in early-type galaxies and show very similar features to those observed in the nuclear regions of HCG62a: the velocity profile is characterized by a central asymmetry, to which corresponds an unusual central isophotal flattening (see Krajnovic et al. 2004).

Similar behavior are observed for instance in NGC 3623, belonging to the Leo Triplet (Afanasiev et al. 2005). The central region of this galaxy, in fact, shows the presence of a chemically distinct core, a relic of a star formation burst, due to interactions, that is shaped as a cold stellar disk with a radius of 250-350 pc. Like NGC 4778, NGC 3623 also shows a drop in the stellar velocity dispersion in the nucleus. Numerical simulations (Bournaud et al. 2003) predict that peculiar nuclear components may be the result of an interaction event between two galaxies. Both major merging and accretion of external material may induce that some gas does flow in to the nuclear regions of the remnant and quickly forms a small concentration of new stars that maintain the original angular momentum of the initial galaxy, counter-rotate with respect to the host galaxy. Furthermore, $N$-body simulations including gas, stars and star formation, suggest that galaxies can develope a central velocity dispersion drop due to nuclear gas inflow, then subsequent star

\footnotetext{
${ }^{2} M_{B}$ is the total magnitude in the $B$ band, derived from the apparent magnitude within the isophote at $2 R_{\mathrm{e}}$.
} 
formation and the appearance of young luminous stars born from dynamically cold gas (Wozniak et al. 2006).

- Formation and evolution. Our data are in good agreement with those obtained by Rampazzo et al. (1998) along the direction connecting the nuclei of NGC 4778 and NGC 4776 $\left(\mathrm{PA}=128^{\circ}\right)$. They also found that the rotation curve is not symmetric with respect to the center of NGC 4778, with a rapid increase in the SE direction at about $15^{\prime \prime}$ from the center. The velocity dispersion increases on both sides, reaching a maximum at about $10^{\prime \prime}$ from the center on the SW side. Given that the close galaxy NGC 4776 is located on the NW, they suggested that $i$ ) the rapid variation of the rotation curve and the sharp increase of the velocity dispersion in the SE direction is a real effect, reliable due to a dynamical perturbation; $i$ ) while the rise towards NGC 4776 (on the NW side) could be partly an artifact due to the apparent superposition of the galaxies. The new kinematics along the SW side (presented in this work) further suggest that the South side of NGC 4778 is dynamically perturbed.

As we have discussed in the Sect. 5, the rotation curve of NGC 4778 is not symmetric with respect to the center, and this is a feature observed in many other compact groups (Bonfanti et al. 1999). According to the literature, many compact groups mainly composed by early-type galaxies, like HCG62, show several morphological signature of interactions, and for all of them the apparent kinematical interactions are not explainable as a mere optical superposition. This conclusion is strongly supported by the simulations performed by Combes et al. (1995), which show that the peculiarities observed in many rotation curves of galaxies belonging to compact groups are due to intrinsic effects and not to contamination along the line of sight. Our results are also in agreement with the estimates coming from merger simulations (Combes et al. 1995), that predict asymmetry in the kinematical profiles and a distinction between the photometric and the dynamical center.

The asymmetry and the shape of the rotation curve and velocity dispersion profile of NGC 4778 do not find correlation with the photometric features of the galaxy, except for the bluer colors in the central region. The absence of correlation between the dynamical and the morphological peculiarities suggests that the dynamical properties of the HCG galaxies may be due to a minor merger event. In fact, as showed by Nishiura et al. (2000), weak galaxy collisions could not perturb the galaxy rotation curves, but morphological deformations could be induced in the outer parts of the galaxy (tidal tails, bridges etc.), while minor mergers could perturb the rotation curves in the inner regions, especially for gas-poor early-type galaxies, without causing morphological peculiarities.

We have estimated the mass-to-light $(M / L)$ ratio of NGC 4778 in order to derive some constraints on the amount of dark matter in HCG62. Since the kinematical profiles are not symmetric, for the calculation of the $M / L$ ratio, we have used the value of $v_{\max }$ and $\sigma_{\max }$ taken from the unperturbed side (NE) of the curves. Moreover, in absence of an accurate photometric calibration, we used as total $B$ magnitude the value provided by NED, $m_{\mathrm{b}}=13.79$. Choosing the values $v_{\max } \simeq 80 \mathrm{~km} \mathrm{~s}^{-1}$, $\sigma_{\max } \simeq 270 \mathrm{~km} \mathrm{~s}^{-1}$ and $R_{\max }=34 \operatorname{arcsec}\left(\simeq 10 \mathrm{kpc} \simeq 2 R_{\mathrm{e}}\right)$, by using the virial theorem $(M / L)_{\text {vir }} \simeq \frac{2 R\left(\sigma^{2}+v^{2}\right)}{(L G)}$, we obtain $M / L \simeq 20.6$. This abnormally high mass-to-light ratio is compatible with a recent merging which has induced a tidal heating in the center of NGC 4778, thus leading to a velocity dispersion which is too high with respect to the actual mass of the galaxy. This result however presents conflicting aspects. In fact, while such behaviour is predicted by numerical simulations
(Combes et al. 1995), a detailed study of the x-ray diffuse halo detected in the central regions of the group leads to a very similar virial estimate of $M / L$. A more detailed discussion of this point will be presented in Sodani et al. (2006).

The velocity dispersion of HCGs are generally higher than would be expected given their visible mass (even if the discordant galaxies are ignored): this can also be explained if the bulk of the mass is in a non visible form (Hickson 1997). Moreover, ROSAT observations revealed a massive hydrogen envelope surrounding HCG62, and showed that this group is dominated by dark matter. Both $N$-body and hydrodynamic simulations indicate that the dark matter halos of individual galaxies merge first, creating a massive envelope within which the visible galaxies move (Barnes 1984; Bode et al. 1993). Kinematic studies of loose groups (e.g. Puche \& Carignan 1991) indicate that the dark matter is concentrated around the individual optical galaxies. In contrast, the X-ray observations indicate that in most compact groups, the gas and dark matter are more extended and are decoupled from the galaxies. This may be consistent with a $M / L 30 \%$ to $50 \%$ lower in compact groups respect to isolated galaxies (Rubin et al. 1991).

The hierarchical mergers of cluster galaxies might power the emission line gas in the center of the group members (Valluri et al. 1996): according to the merger scenario, in order to power emission-line nebulae, the merger must include a galaxy or a group of galaxies that are late-types and which bring with them

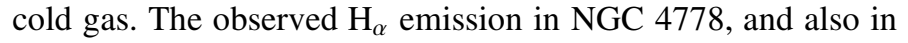
NGC 4776 and NGC 4761, further support the idea that this galaxy has recently experienced a merger event.

The overall scenario depicting NGC 4778 as the product of a recent merger, as emerges by previous discussion, is consistent with the results obtained from X-ray observations. The presence of an extended X-ray halo is consistent with scenarios describing current compact group as the result of a first generation of mergers, where the dominant galaxy sits at the bottom of a large common gravitational well. The presence of two X-ray cavities in the hot gaseous halo located on symmetrically with respect to NGC 4778 also indicate that the AGN residing in the galaxy core, must have undergone a recent (a few $10^{7} \mathrm{yr}$, Birzan et al. 2004) active fase during which the radio-emitting relativistic plasma has created two low density regions within the hot IGM. It is commonly believed that such activity can be triggered by merging events, which increases the accretion rate onto the central massive black hole (Cattaneo et al. 2005). These fits a scenario in which NGC 4778 underwent a merger sometime in the past which produced the counter-rotating core and triggered the nuclear activity. However the low incidence of strong, type I AGN activity in interacting galaxies suggests that a delay of several $10^{8}$ years is generally expected until the peak of the AGN fase (Canalizo et al. 2006; Grogin et al. 2005, however see also Koulouridis et al. 2006). In the case of NGC 4778 we can derive a lower limit of $10^{7} \mathrm{yr}$ for such delay from the age of the cavities. On the other end, an upper limit is represented by the age of the merger which, given the typical dynamical timescales of Compact Groups, can be estimated in $10^{8} \mathrm{yr}$. This result agrees with the estimate that AGNs have duty cycles of the order of $10^{7-8}$ years.

Acknowledgements. The authors very grateful to R. Saglia for making available the software for the kinematical study of early-type galaxies. M.S. also wishes to thank the INAF-Observatory of Capodimonte for the hospitality given during her thesis work, and N. Napolitano for many useful discussions and suggestions. This work was funded through a grant from Regione Campania (ex legge 5) and a MIUR grant. This work is based on observations made with ESO Telescopes at the Paranal Observatories under programme ID $\langle 169 . A-0595(C)\rangle$ and $\langle 169 . A-$ $0595(D)\rangle$. 


\section{References}

Afanasiev, V. L., \& Sil'chenko, O. K. 2005, A\&A, 429, 825

Balcells, M., \& Gonzalez, C. G. 1998, ApJ, 505, 109

Barnes, J. 1984, MNRAS, 208, 873

Bender, R. 1990, A\&A, 229, 441

Bender, R., Burstein, D., \& Faber, S. M. 1992, ApJ, 399, 462

Bender, R., Saglia, R. P., \& Gerhard, O. E. 1994, MNRAS, 269, 785

Bertola, F., \& Corsini, E. 1999, Galaxy Interactions at Low and High Redshift, ed. J. E. Barnes, \& D. B. Sanders, Proc. of IAU Symp., 186, 149

Bettoni, D., Buson, L. M., Maira, L., \& Bertola, F. 1995, ASP Conf. Ser., 70, 95

Binney, J. 1978, MNRAS, 183, 501

Binney, J. 1985, MNRAS, 212, 767

Birzan, L., Rafferty, D. A., McNamara, B. R., et al. 2004, ApJ, 607, 800

Bode, P. W., Cohn, H. N. \& Lugger, P. M. 1993, ApJ, 416, 17

Bonfanti, P., Simien, F., Rampazzo, R., \& Prugniel, Ph. 1999, A\&AS, 139, 483

Bournaud, F., \& Combes, F. 2003, A\&A, 401, 817

Byun, Y. I \& Freeman, K. C. 1995, ApJ, 448, 563

Canalizo, G., Stockton, A., Brotherton, M. S., et al. 2006, Proc. of the QSO Host Galaxies: Evolution and Environment, Conf. Lorentz Center, Universiteit Leiden, August 2005; 2006astro.ph..3218C

Caon, N., Capaccioli, M., \& D’Onofrio, M. 1993, MNRAS, 265, 1013

Cattaneo, A., Combes, F., Colombi, S., et al. 2005, MNRAS 359, 1237

Combes, F., Rampazzo, R., Bonfanti, P. P., Prugniel, P., \& Sulentic, J. W. 1995, A\&A, 297, 37

Coziol, R., Ribeiro, A. L. B., De Carvalho, R., \& Capelato, H. V. 1998, ApJ, 493, 563

Eke, V. R., Frenk, C. S., Baugh, C. M., et al. 2004, MNRAS, 355, 769

Freeman, K. C. 1970, ApJ, 160, 811

Fukazawa, Y., Nakazawa, K., Isobe, N., Ohashi, T., \& Kamae, T. 2001, ApJ, 546, 87

Fukugita, M., Shimasaku, K., \& Ichikawa, T. 1995, PASP, 107, 945
Gerhard, O. E. 1993, MNRAS, 265, 213

Governato, F., Bhatia, R., \& Chincarini, G. 1991, ApJ, 371, 15

Grogin, N. A., Conselice, C. J., Chatzichristou, E., et al. 2005, ApJ, 627, L97

Hickson, P., 1997, ARA\&A, 35, 357

Hickson, P., Kindl, E. \& Huchra, J. P. 1988, ApJ, 331, 64

Iodice, E., D’Onofrio, M., \& Capaccioli, M. 2001, Ap\&SS, 276, 869

Jog, C. J., \& Maybhate, A. 2006, MNRAS, 000, 000

Koulouridis, E., Plionis, M., Chavushyan, V., et al. 2006, ApJ, 639, 37

Krajnovic, D., \& Jaffe, W. 2004, A\&A, 428, 877

Kropolin, W., \& Zeilinger, W. W. 2000, A\&AS, 145, 71

Mehlert, D., Saglia, R., Bender, R., \& Wegner, G. 2000, A\&AS, 141, 449

Mendes de Oliveira, C. 1992, Ph.D. Thesis, Univ. of British Columbia

Mendes de Oliveira, C., Aram, P., Plana, H., \& Balkowski, C. 2003, AJ, 126, 2635

Nishiura, S., Shimada, M., Ohyama, Y., Murayama, T., \& Taniguchi, Y. 2000, AJ, 120, 1691

Pildis, R. A., Evrard, A. E., \& Bergman, J. N. 1996, AJ, 112, 378

Puche, D. \& Carignan, C. 1991, ApJ, 378, 487

Rampazzo, R., Covino, S., Trinchieri, G., \& Reduzzi, L. 1998, A\&A, 330, 423

Rood, H. J. \& Struble, M. F. 1994, PASP, 106, 416

Rubin, V. C., Hunter, D. A., \& Ford, W. K. Jr. 1991, ApJS, 76, 153

Sodani, S., De Filippis, E., Paolillo, M., Longo, G., \& Spavone, M. 2006, in preparation (Paper II)

Tovmassian, H. M. 2001, PASP, 113, 543

Tully, R. B., \& Fisher, J. R. 1987, S\&T, 74, 612

Valluri, M., \& Anupama, G. C. 1996, AJ, 112, 1390

Vennik, J., Richter, G. M., \& Longo, G. 1993, AN, 314, 393

Vrtilek, J. M., Grego, L., David, L. P., et al. 2002, APS meeting, B17.107

Wozniak, H., \& Champavert, N. 2006, MNRAS, 000, 000

Zabludoff, A. I., \& Mulchaey, J. S. 1998, ApJ, 498, L5

Zepf, S. E., Whitmore, B. C., \& Levison, H. F. 1991, ApJ, 383, 524 